\title{
558: Calculation of Eddy Currents in the ETE Spherical Torus
}

\author{
G.O. Ludwig \\ Instituto Nacional de Pesquisas Espaciais, 12227-010 S. J. Campos, SP, Brazil
}

\begin{abstract}
The currents induced during startup in the vacuum vessel of the ETE spherical torus (Experimento Tokamak Esférico) are evaluated using a circuit model based on the Green's function method. The distribution of eddy currents is calculated using a thin shell approximation for the vessel and local curvilinear coordinates. The predicted results agree quite well with values of the eddy currents measured in ETE.
\end{abstract}

\section{INTRODUCTION}

This paper presents a magnetostatic model developed to evaluate the currents induced during startup in the continuous vacuum vessel of the ETE spherical torus. The distribution of eddy currents is modeled using a thin shell approximation for the vessel. The equation governing the induction of surface current on the thin shell is derived using the Green's function method. Symmetry considerations, and adoption of both a local curvilinear coordinates system and a spectral representation for the contour of the vacuum vessel reduce this three-dimensional problem in space to one dimension. The resulting one-dimensional integral equation for the surface current can be solved expanding the current in a Fourier series in the poloidal angle. Finally, by the introduction of Laplace transformation in time, the problem for the set of Fourier components of the surface current is reduced to a circuit model that can be solved by matrix procedures. The results are compared with preliminary measurements of the eddy currents in ETE.

\section{FORMULATION OF THE MAGNETOSTATIC PROBLEM}

The surface current density in a thin shell of thickness $\delta$ is given in terms of the current density by $\vec{K}=\delta \vec{j}$ [1], where the current density is related to the electric field by Ohm's law, $\vec{j}=\sigma \vec{E}$. Application of Faraday's law for a constant conductivity $\sigma$ leads to

$$
\nabla \times \vec{K}=\sigma \delta \nabla \times \vec{E}=-\sigma \delta(\partial \vec{B} / \partial t)
$$

where $\vec{B}$ corresponds to the total induction. The condition of current continuity gives $\nabla \cdot \vec{K}=\sigma \delta \nabla \cdot \vec{E}=0$.

For an axisymmetric configuration the problem is independent of the toroidal angle $\zeta$. Furthermore, the variation of the toroidal flux in time, $\partial \Phi_{T} / \partial t$, is neglected during startup, and so no poloidal currents are induced on the vacuum vessel. In this case, axisymmetry and the solenoidal property of the magnetic field, $\nabla \cdot \vec{B}=0$, imply a single toroidal component of the vector potential. In vector form the potential is given in terms of the poloidal flux $\Phi_{P}$ by $\vec{A}=(2 \pi)^{-1} \Phi_{P} \nabla \zeta$. In the same way, the surface current vector is expressed in terms of the single toroidal component $K_{T}$ by $\vec{K}=h_{\zeta} K_{T} \nabla \zeta$, where the scale factor $h_{\zeta}=|\partial \vec{r} / \partial \zeta|$ corresponds to the radial distance to the symmetry axis in cylindrical coordinates. Now, the magnetic induction is calculated in terms of the poloidal flux by

$$
\vec{B}=\nabla \times \vec{A}=-(2 \pi)^{-1} \nabla \zeta \times \nabla \Phi_{P} .
$$

This equation, combined with the previous one and the assumption of an uniform distribution over the small thickness $\delta$, leads to a relation between the toroidal surface current density and the local value of the poloidal flux:

$$
K_{T}=-\sigma \delta\left(2 \pi h_{\zeta}\right)^{-1}\left(\partial \Phi_{P} / \partial t\right) \text {. }
$$

In general, the vector potential at any point $\vec{r}$ not lying on the surface $S^{\prime}$ is given by the extension of the BiotSavart law

$$
\vec{A}(\vec{r})=\frac{\mu_{0}}{4 \pi} \iint_{S^{\prime}} \frac{\vec{K}\left(\vec{r}^{\prime}\right)}{\left|\vec{r}-\vec{r}^{\prime}\right|} d^{2} r^{\prime}+\vec{A}_{\text {ext }}(\vec{r}),
$$


where $\vec{A}_{\text {ext }}$ stands for the external sources. The differential element of area in the coordinate surface $\rho$ that coincides with the surface layer of current is $d^{2} r(\rho)=h_{\zeta} d \ell(\theta) d \zeta$. Using the properties $|\nabla \zeta|^{2}=h_{\zeta}^{-2}$ and $\nabla \zeta \cdot \nabla \zeta^{\prime}=\cos \left(\zeta-\zeta^{\prime}\right) /\left(h_{\zeta} h_{\zeta^{\prime}}\right)$ the equivalent integral relation for the flux function is

$$
\Phi_{P}(\vec{r})=\mu_{0} \oint K_{T}\left(\vec{r}^{\prime}\right)\left\langle\frac{\pi h_{\zeta} h_{\zeta^{\prime}} \cos \left(\zeta-\zeta^{\prime}\right)}{\left|\vec{r}-\vec{r}^{\prime}\right|}\right\rangle_{\zeta^{\prime}} d \ell\left(\theta^{\prime}\right)+\Phi_{e x t}(\vec{r}),
$$

where $\langle\ldots\rangle_{\zeta}=(2 \pi)^{-1} \int(\ldots) d \zeta$. This defines the Green's function for the axisymmetric Ampère's law, $G\left(\vec{r}, \vec{r}^{\prime}\right)=\left\langle\pi h_{\zeta} h_{\zeta^{\prime}} \cos \left(\zeta-\zeta^{\prime}\right) /\left|\vec{r}-\vec{r}^{\prime}\right|\right\rangle_{\zeta^{\prime}}$. The Green's function integral for $\Phi_{P}$ automatically satisfies the boundary condition $\widehat{n} \cdot \nabla \Phi_{P}=-2 \pi \mu_{0} h_{\zeta} K_{T}$, which corresponds to the discontinuity of the magnetic induction across the surface layer of current, $\widehat{n} \times[\vec{B}]_{S}=\mu_{0} \vec{K}$ ( $\widehat{n}$ is the unit normal). Finally, taking the derivative with respect to time and using the relation between $K_{T}$ and $\partial \Phi_{P} / \partial t$ provided by Faraday's law, the excitation of Foucault currents in a thin axisymmetric shell is governed by the equation

$$
\frac{2 \pi h_{\zeta}}{\sigma \delta} K_{T}(\vec{r})=-\mu_{0} \oint \frac{\partial K_{T}\left(\vec{r}^{\prime}\right)}{\partial t} G\left(\vec{r}, \vec{r}^{\prime}\right) d \ell\left(\theta^{\prime}\right)-\frac{\partial \Phi_{e x t}(\vec{r})}{\partial t} .
$$

This equation has local terms depending on the shell resistivity and non-local terms depending on mutual inductance effects between diverse regions of the current distribution. The total toroidal current induced in the shell is $I_{T}=\int_{0}^{2 \pi} K_{T}(\theta) h_{\theta} d \theta$, where the scale factor $h_{\theta}=|\partial \vec{r} / \partial \theta|$.

\section{SPECTRAL REPRESENTATION OF THE ETE VACUUM VESSEL}

In order to apply effectively the one-dimensional integral equation for the eddy currents obtained in the previous section, it is necessary to use a coordinate system coinciding with the contour of the axisymmetric shell. The centerline of the ETE vacuum vessel has an exact sectionally (piecewise) continuous representation shown on the left side of Figure 1 as a continuous line.
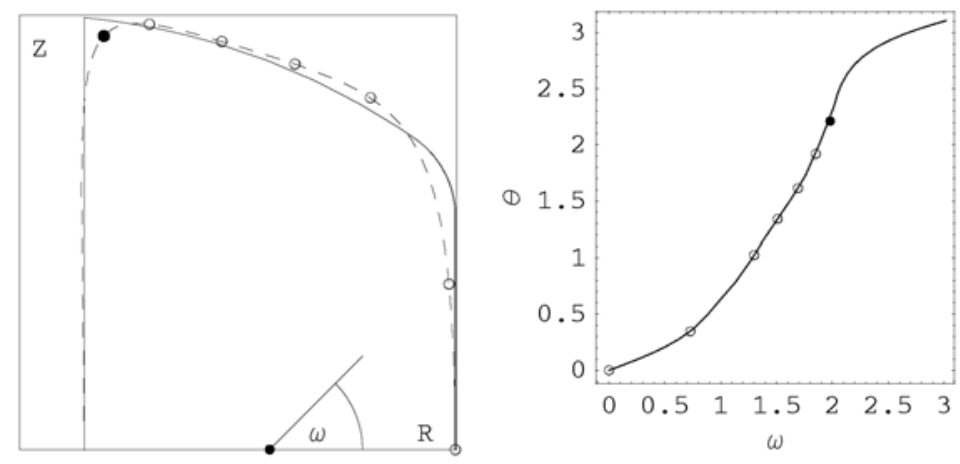

FIGURE 1. The centerline of the ETE vacuum vessel (continuous line) and its spectral fit (dashed line) are shown on the left side. The white circles correspond to the center of flanges used to measure the eddy current distribution, and the black circle on the spectral fit corresponds to the inner edge of the vessel centerline. The adjusted $\theta-\omega$ mapping is shown on the right side.

In accordance with Figure 1 the sectional continuous representation specifies the cylindrical coordinates $R(\omega)$, $Z(\omega)$ as functions of the poloidal angle $\omega$ in a pseudo-toroidal coordinate system centered in the cross-section of the vacuum vessel. Now, the centerline of the vacuum vessel can be represented approximately by a truncated spectral expansion in Chebyshev polynomials:

$$
\left\{\begin{array}{l}
R(\theta)=C_{0}+C_{1} \cos \theta-a \sum_{n=1}^{N} C_{n}\left[1-T_{n}(\cos \theta)\right] \\
Z(\theta)=E_{V} \sin \theta\left[C_{1}-a \sum_{n=1}^{N} C_{n} U_{n-1}(\cos \theta)\right]
\end{array}\right.
$$

The coefficients $C_{0}$ and $C_{1}$ are determined by the constraints $R(0)=R_{0}+a, R(\pi)=R_{0}-a$, where $R_{0}$ and 
$a$ are the major and minor radii of the toroidal vessel, respectively. The elongation $E_{V}$ and the remaining spectral coefficients $C_{2}, C_{3}, \ldots C_{N}$ can be determined by a least-squares fitting procedure. In the case of the ETE vacuum vessel a reasonable spectral representation can be obtained including only elongation, triangularity and quadrangularity (squareness) corrections. The least-squares calculation gives $E_{V}=2.164, C_{0}=0.320, C_{1}=0.287, C_{2}=0.0981$ and $C_{3}=-0.110$, and the resulting spectral fit is show on the left side of Figure 1 as a dashed line. The least-squares fitting procedure includes also a determination of the best mapping between the pseudo-toroidal angle coordinate $\omega$ and the poloidal angle $\theta$ in the local curvilinear coordinate system. The adjusted $\theta-\omega$ mapping is shown on the right side of Figure 1.

\section{FOURIER COMPONENTS OF THE SURFACE CURRENT}

The integral equation (1) for the Foucault currents in a thin shell that was derived in the second section of this paper can be solved by expansion of $K_{T}(\theta, t)$ in a Fourier series

$$
K_{T}(\theta, t)=\frac{1}{2 \pi h_{\theta}(\theta)}\left(I_{T}(t)+\sum_{n=1}^{\infty} I_{n}(t) \cos n \theta\right) .
$$

The total toroidal current flowing in the axisymmetric shell is $I_{T}(t)$ according to the definition in the second section. Substitution of the Fourier series (2) in the integral equation (1) gives

$$
\frac{h_{\zeta}(\theta)}{\sigma \delta h_{\theta}(\theta)}\left(I_{T}(t)+\sum_{n=1}^{\infty} I_{n}(t) \cos n \theta\right)=-\mu_{0}\left(\frac{\partial I_{T}}{\partial t}\left\langle G\left(\theta, \theta^{\prime}\right)\right\rangle_{\theta^{\prime}}+\sum_{n=1}^{\infty} \frac{\partial I_{n}}{\partial t}\left\langle G\left(\theta, \theta^{\prime}\right) \cos n \theta^{\prime}\right\rangle_{\theta^{\prime}}\right)-\frac{\partial \Phi_{e x t}}{\partial t}
$$

where $\langle\ldots\rangle_{\theta}=(2 \pi)^{-1} \int(\ldots) d \theta$. Limiting the Fourier coefficients to order $\ell$, the $\cos m \theta$ harmonics of this equation result in a set of $\ell+1$ linear equations for $I_{T}(t)$ and $I_{n}(t)$ that can be written in the form

$$
R_{0 m} I_{T}(t)+L_{0 m} \frac{\partial I_{T}}{\partial t}+\sum_{n=1}^{\ell}\left(R_{n m} I_{n}(t)+L_{n m} \frac{\partial I_{n}}{\partial t}\right)=-\frac{\partial}{\partial t}\left\langle\Phi_{e x t}(\theta, t) \cos m \theta\right\rangle_{\theta},
$$

where $R_{n m}$ and $L_{n m}$ are resistance and mutual inductance coefficients defined by:

$$
\left\{\begin{aligned}
R_{n m} & =\frac{1}{\sigma \delta}\left\langle\frac{h_{\zeta}(\theta)}{h_{\theta}(\theta)} \cos n \theta \cos m \theta\right\rangle_{\theta} \\
L_{n m} & =\mu_{0}\left\langle\left\langle G\left(\theta, \theta^{\prime}\right) \cos n \theta^{\prime}\right\rangle_{\theta^{\prime}} \cos m \theta\right\rangle_{\theta}
\end{aligned}\right.
$$

These definitions and the symmetry of the Green's function show that $R_{n m}$ and $L_{n m}$ are symmetric matrices.

In general, the external flux is the sum of the magnetizing flux $\Phi_{M}(t)$ produced by an ideal transformer and the fluxes $\Phi_{k}(\vec{r}, t)$ produced by sets of poloidal field coils:

$$
\Phi_{\text {ext }}(\vec{r}, t)=\Phi_{M}(t)+\sum_{k} \Phi_{k}(\vec{r}, t)=\Phi_{M}(t)+\mu_{0} \sum_{k} I_{k}(t)\left[G_{k}\left(\vec{r}, R_{k}, Z_{k}\right)+G_{k}\left(\vec{r}, R_{k},-Z_{k}\right)\right]
$$

where it was assumed that the external coils are formed by pairs of coils placed symmetrically with respect to the equatorial plane, and connected in series. Defining the mutual inductance coefficients, $L_{k m}=$ $\mu_{0}\left\langle\left[G_{k}(\theta)+G_{k}(-\theta)\right] \cos m \theta\right\rangle_{\theta}$, the equations for the Fourier coefficients of the surface current density may be written $\left(\delta_{n m}\right.$ is the Kronecker delta)

$$
R_{0 m} I_{T}(t)+L_{0 m} \frac{\partial I_{T}}{\partial t}+\sum_{n=1}^{\ell}\left(R_{n m} I_{n}(t)+L_{n m} \frac{\partial I_{n}}{\partial t}\right)=-\frac{\partial \Phi_{M}}{\partial t} \delta_{0 m}-\sum_{k} L_{k m} \frac{\partial I_{k}}{\partial t} .
$$

In this way the problem of Foucault currents induced in a thin axisymmetric shell is reduced to the solution of a set of circuit-like coupled linear equations for the Fourier components of the surface current density. The calculation of the mutual coefficients $L_{n m}$ requires some attention due to self-field effects related to the singular character of the Green's function [2].

\section{SOLUTION OF THE CIRCUIT MODEL AND RESULTS}

It is now an easy matter to solve the set of circuit equations (3) for the Fourier components of the Foucault current. 
Introducing Laplace transformation in time and denoting the complex frequency by $s$, the equations for $I_{T}(s)$ and $I_{n}(s)(n=1,2, \ldots, \ell)$ can be written in matrix form, $[[R+s L]][I(s)]=-s[\Phi(s)]$, where $[[R+s L]]$ is a symmetric matrix. The initial values of the magnetizing sources are taken equal to zero at startup. The solution of the circuit model is obtained simply by multiplying the vector of electromotive forces $-s[\Phi(s)]$ by the inverse matrix $[[R+s L]]^{-1}$ and then calculating the inverse Laplace transform. One advantage of the method is that the inverse matrix depends only on the geometry of the problem, which is independent of the detailed excitation.

The resistance and inductance components scale as $A /\left(E_{V} \sigma \bar{\delta}\right)$ and $\mu_{0} R_{0}[\ln (8 A)-2]$, respectively, where $E_{V}=2.164$ is the elongation, $A=1.346$ is the aspect ratio and $R_{0}=0.348 \mathrm{~m}$ is the major radius of the vacuum vessel. The conductivity of Inconel at room temperature is $\sigma \cong 7.8 \times 10^{5}(\Omega \cdot \mathrm{m})^{-1}$. Now, the thickness of the vacuum vessel is $\Delta_{V}=6.35 \mathrm{~mm}$ for both the torispherical head and the external cylindrical wall, and $\delta_{V}=1.00 \mathrm{~mm}$ for the internal cylindrical wall. In the calculation of $R_{n m}$ the $\theta$ integration is split in two sections to account for the change in the wall thickness, and an average thickness $\bar{\delta} \cong 4.59 \mathrm{~mm}$ is defined taking into account the length of the two segments. The average surface current scales as $\bar{K}_{T} \sim A I_{T} /\left(2 \pi R_{0}\right)$.

Calculations of the eddy current behavior in space and time were performed and compared with measurements taken in the ETE vacuum vessel. Satisfactory results were obtained including only three harmonics, $\ell=3$, in the calculations. Figure 2 shows the distribution of the surface current at the instant $\tau_{0} / 4$ that corresponds approximately to the maximum negative value of the induced current $\left(\tau_{0}=\mu_{0} \sigma \bar{\delta} R_{0} / A \cong 1.16 \mathrm{~ms}\right.$ sets the time scale). From the plot in Figure 2 and the mapping $\theta-\omega$ shown in Figure 1 one verifies that the eddy current distribution has a peak at $\omega \sim 113^{\circ}, \theta \sim 127^{\circ}$, near the inside corner of the vacuum vessel contour and in accordance with rough measurements of the distribution excited by the ohmic heating system in ETE. The bar chart in Figure 2 compares the relative currents measured over several sectors of the vacuum chamber (light gray bars) with the calculated values (dark bars).
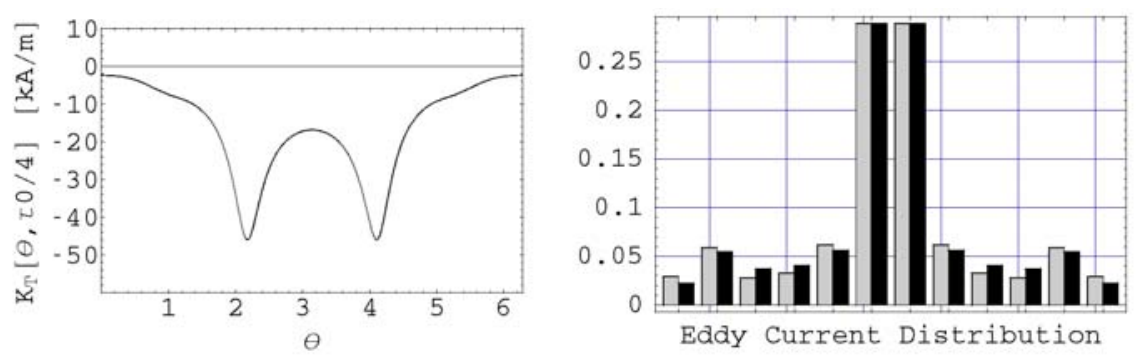

FIGURE 2. The calculated eddy current distribution in the vacuum vessel is shown on the left at the instant of maximum current induced during tests. The measured and calculated distributions are compared on the right.

Based on these results the operation of a pair of compensation coils is being optimized to apply a vertical field bias during plasma breakdown in ETE. In addition, the eddy current distribution is being used to model the vacuum vessel effects in plasma discharge simulations during the early phase. In these zero-dimensional simulations the external inductance of the low aspect ratio ETE plasma and the mutual inductance coefficients between the plasma, the vacuum vessel and the external poloidal field coils are calculated in accordance with a previous work [3].

\section{ACKNOWLEDGMENTS}

This work was partially supported by The State of São Paulo Research Foundation (FAPESP) and by the International Atomic Energy Agency (IAEA).

\section{REFERENCES}

1. J.A. Stratton, Electromagnetic Theory, McGraw-Hill, New York, 1941.

2. S.P. Hirshman and G.H. Neilson, Phys. Fluids, 29, 790 (1986).

3. G.O. Ludwig and M.C.R. Andrade, Phys. Plasmas, 5, 2274 (1998). 